\title{
Increased Microanalysis Lab Productivity: Automation, Integration and Speed
}

\author{
N.A. Bulloss
}

Thermo Fisher Scientific, 5225 Verona Rd, Madison, WI 53711

Electron microscope time is expensive; most industrial labs want to get the maximum number of samples through the lab (with the right answers to their questions!) in the minimum amount of time. Advances in x-ray detectors, electronics and software have allowed significant increases in sample throughput in the microanalysis laboratory. This efficiency can be achieved through reducing the learning curve for the user, automating many commonly used aspects of analysis, faster detectors, advanced data processing and seamless integration of detectors within the system.

Modern energy-dispersive spectroscopy (EDS) silicon drift detectors (SDD) allow operators to collect x-rays at very high count rates with little degradation in resolution or peak shift. Commonly operators are concerned about taking advantage of the higher count rates and abilities to collect and analyze data at high dead times. We will show how the high throughput silicon drift detectors can be used to significantly reduce acquisition times and increase sample throughput. In addition we will demonstrate the consistency in quantitative analysis across a range of detector dead times.

Recent advances in WDS technology have brought the ease of use of WDS to parallel that of EDS. With accompanying advances in motor technology and hardware design, WDS analyses can be performed at hitherto unattainable speeds. By closely integrating and automating both EDS and WDS analyses, it is possible to confirm EDS peak identification using WDS during x-ray collection. This is of particular use when analyzing samples with potential peak overlaps, e.g. Mo L $\alpha$ and S K $\alpha$, and transition element $\mathrm{L}$ lines ( $\mathrm{Fe} \mathrm{L} \alpha, \mathrm{Cr} \mathrm{L} \alpha$ and $\mathrm{Ni} \mathrm{L} \alpha$ ) when operating at low accelerating voltages. The EDS spectrum is used to identify potential peak overlaps, the WDS then automatically scans over the potential overlaps to confirm EDS peak identifications. WDS can be run standalone or combined with EDS when performing quantitative analyses. By using WDS for quantitative analysis only on those elements with low concentrations, peak overlaps or whenever a larger peak to background ratio is needed, analysis times can be significantly reduced.

Software has advanced far beyond simple elemental gross count $\mathrm{x}$-ray mapping. Element maps require post collection analysis, particularly when peak overlaps exist in the sample. To mitigate errors in interpretation of samples with peak overlaps, we perform quantitative mapping of spectral imaging data sets. In quantitative mapping, the spectrum at every pixel undergoes the same background removal, peak deconvolution, and matrix corrections as traditional spectral analyses. The maps can then be quantified and displayed as net counts, weight percent, atomic percent etc. These maps now provide the correct spatial distribution of the elements within the sample. Taking mapping one step further is phase or component mapping performed during data acquisition. Phase mapping software employs multivariate statistical analysis routines to identify chemical components or phases in the sample. Multi-variate analysis software can use sparse or low volume x-ray data to determine chemically unique phases in a sample. The advantage of being able to analyze using such small amounts of data is that it further reduces the analysis time required to achieve results. Additionally, each operator gets the same answer, therefore errors or differences in data interpretation are avoided. Figures 1 and 2 show how a sample with peak overlaps (in this example Mo L $\alpha$ and $S \mathrm{~K} \alpha$ ) that provide very little contrast in gross count elemental maps can be uniquely phase mapped thanks to a robust peak deconvolution routine in just a few minutes. 

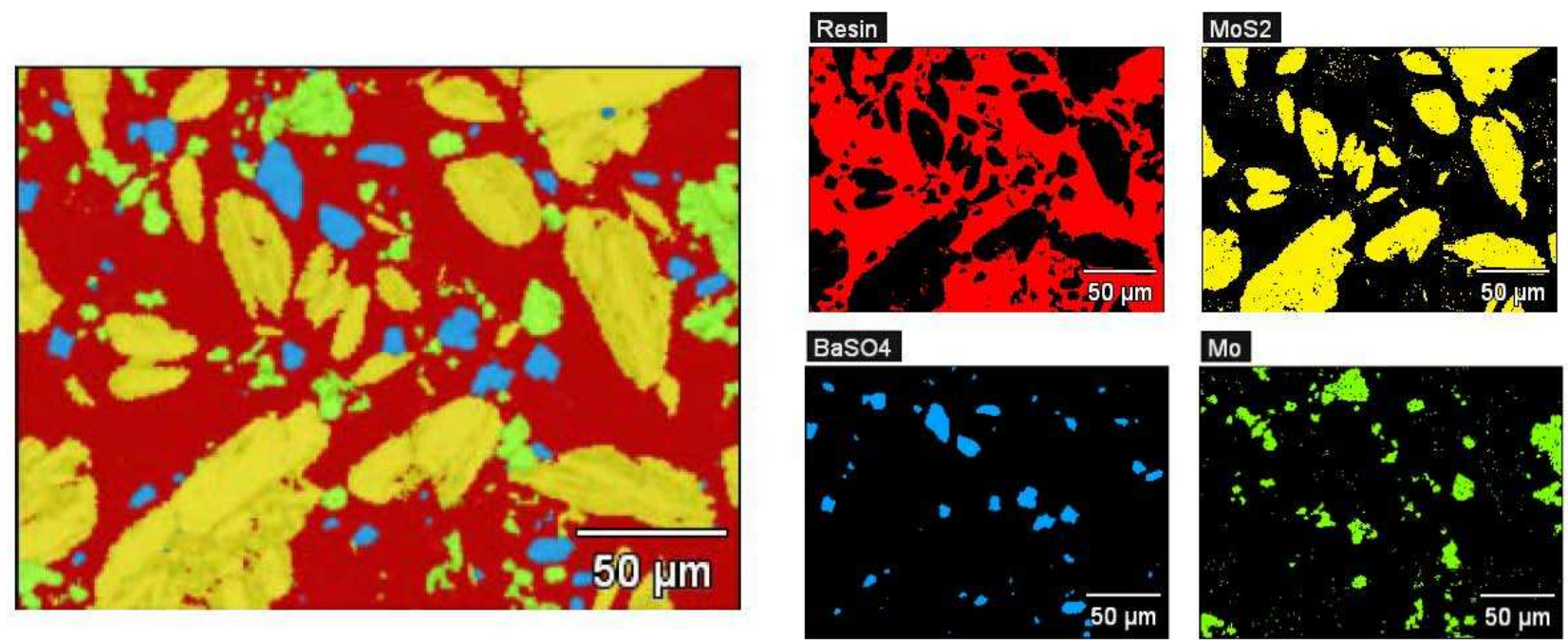

FIG. 1. Phase map of a Mo, S, Ba O sample collected using a SDD detector at $7 \mathrm{kV}$. The total collection/analysis time for this sample was 3 minutes.

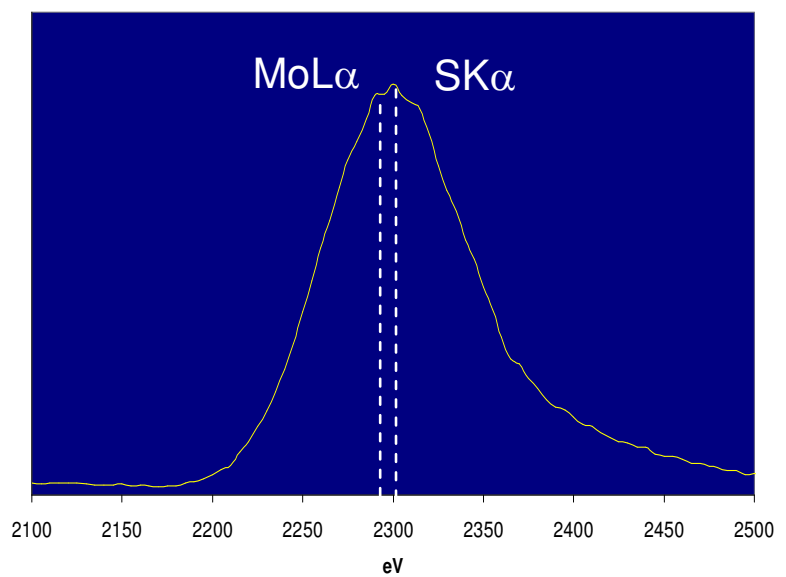

FIG. 2. Simulated EDS spectra from the $129 \mathrm{eV}$ SDD used in figure 1. The phase software performs peak deconvolution to clearly distinguish between the Mo and $\mathrm{S}$. 